\section{Pericecal abscess treated by endoscopic transappendiceal stenting}

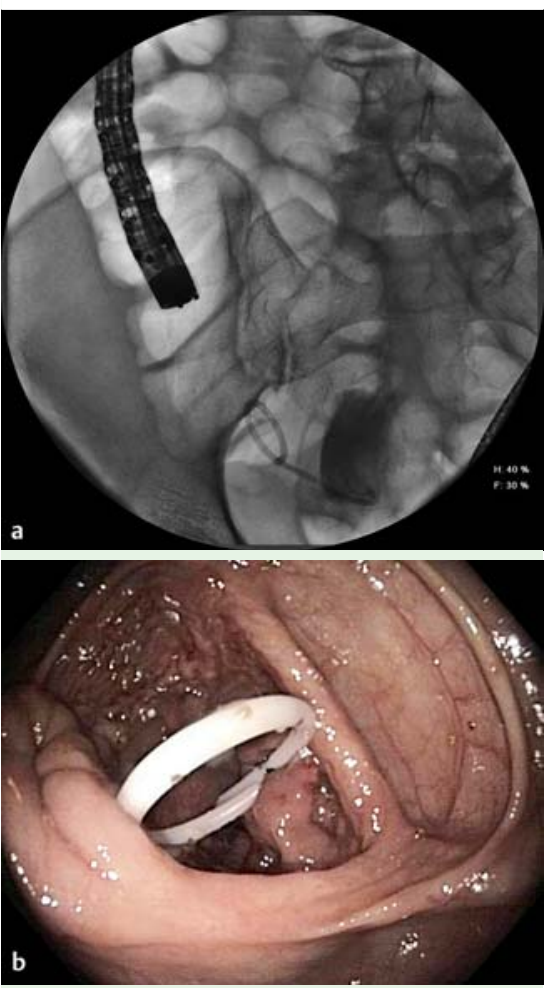

Fig. 4 a, b A 4-cm long, 7-Fr double pigtail catheter placed in the abscess cavity.

Al we intubated the appendix easily, using a guide wire sphincterotome, and plenty of pus discharged from the appendiceal orifice ( $\bullet$ Fig. 2). The abscess was visualized on fluoroscopy, using a contrast medium (๑ Fig.3). A double pigtail catheter, $4 \mathrm{~cm}$ in length and 7Fr in diameter, was placed into the abscess cavity ( $\bullet$ Fig. 4 ). The patient was free of pain immediately after drainage and CRP levels returned to normal within a few days. Ultrasonographic follow-up demonstrated rapid resolution of the abscess. An abdominal X-ray taken 3 weeks later showed that the pigtail catheter was no longer in place but had passed spontaneously. The consulted surgeon did not carry out interval appendectomy since no further episodes of appendicitis were expected to occur because of the likely obliteration of the appendiceal lumen [4]. After 6 months follow-up the patient is well without any recurrence or clinical signs of appendicitis.

Percutaneous drainage in conjunction with antibiotic therapy is the treatment of choice in patients with pericecal abscesses. In this report we have demonstrated that endoscopic transappendiceal drainage and stenting may be an alternative method of management in selected patients. Routine interval appendectomy after successful nonoperative treatment is not generally recommended [4].

Endoscopy_UCTN_Code_TTT_1AQ_2AJ

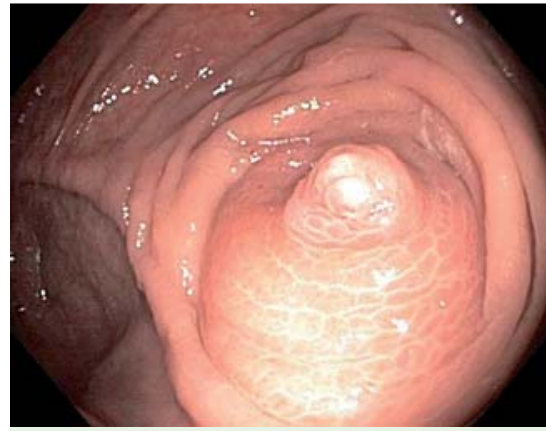

Fig. 1 Colonoscopic view in a 63-year-old woman with abdominal pain but no fever or diarrhea showing the elevated, inflamed appendiceal orifice.

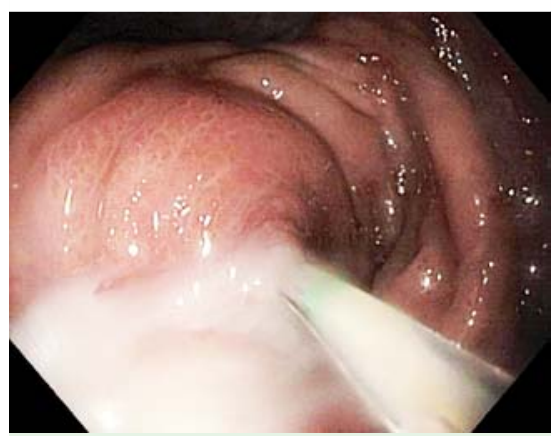

Fig. 2 Profuse discharge of pus from the appendiceal orifice.

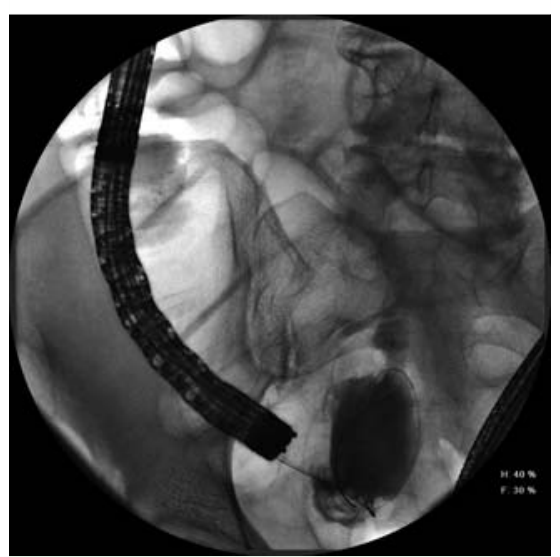

Fig. 3 Fluoroscopic view of the pericecal abscess with guide wire in place.

Competing interests: None

\section{Kapral, F. Wewalka, \\ A. Ziachehabi, R. Schoefl}

Department of Gastroenterology,

Elisabethinen Hospital, Linz, Austria
Bibliography

Dol http://dx.doi.org/

10.1055/s-0032-1309717

Endoscopy 2012; 44: E310

(c) Georg Thieme Verlag KG

Stuttgart · New York

ISSN 0013-726X

Corresponding author

C. Kapral

Department of Gastroenterology

Elisabethinen Hospital

Fadingerstrasse 1

A-4020 Linz, Austria

christine.kapral@elisabethinen.or.at 